\title{
EL CONTEXTO ACADÉMICO COMO FACTOR DIFERENCIADOR EN LA TRANSICIÓN A LA UNIVERSIDAD
}

\author{
O CONTEXTO ACADÊMICO COMO FATOR DIFERENCIADOR NA TRANSIÇÃO PARA A \\ UNIVERSIDADE
}

ACADEMIC CONTEXT AS A DIFFERENTIATING FACTOR IN TRANSITION TO UNIVERSITY

Pilar Figuera Gazo

Doutora em Pedagogia pela Universidad de Barcelona. Docente da Faculdade de Pedagogia da Universidad de Barcelona.

Mercedes Torrado Fonseca Doutora em Pedagogia pela Universidad de Barcelona. Docente da Faculdade de Pedagogia da Universidad de Barcelona.

Departamento de Métodos de Investigação e Diagnóstico em Educação Faculdade de Pedagogia Universidad de Barcelona (UB)

Barcelona - Espanha

Endereço:

Campus Mundet.

Departamento MIDE. Edificio Llevant 2n planta. Paseo Vall Hebron, 171, 08035

Barcelona

E-mail:

pfiguera@ub.edu

\section{RESUMO}

Os estudos sobre transição à universidade destacam a importância de analisar o processo de adaptação no primeiro ano a partir de estudos longitudinais contextualizados. Seguindo essa premissa, o artigo analisa, por um lado, a evolução e os resultados das pesquisas realizadas nos últimos anos sobre a transição à universidade dos estudantes no sistema universitário catalão e, especificamente, da Universidade de Barcelona; e por outro lado, apresenta a última pesquisa desenvolvida em dois âmbitos disciplinares distintos de Ciências Sociais: Administração e Direção de Empresas (ADE) e Pedagogia[1]. Os resultados permitem contextualizar as dificuldades no processo de transição e a importância significativa da adaptação acadêmica no primeiro ano de estudos de maneira segmentada. Finalmente enfoca a necessidade de revisar as medidas de retenção estabelecidas na universidade, incluindo as ações de orientação, com a finalidade de otimizar a persistência dos estudantes.

PALAVRAS-CHAVE: Estudantes universitarios. Adaptação acadêmica. Persistência.

ABSTRACT

Studies on the transition to university stress the importance of using contextualized longitudinal approaches to analyse students' adaptation processes in the first year. This article analyses the evolution and results of research conducted in recent years inside the Catalan university system, and more specifically at the 
University of Barcelona. It also outlines the latest research into the transition to university in two different disciplines in the social sciences: business administration and management, and teacher training. The results provide a contextualized view of the difficulties in the transition process and highlight the importance of academic adjustment in the first year of study. Finally we underline the need to review the measures used at universities to promote student retention, such as orientation activities.

KEYWORDS: University students. Academic adaptation. Persistence.

\section{RESUMEN}

Los estudios sobre la transición a la universidad destacan la importancia de analizar el proceso de adaptación en el primer año a partir de estudios longitudinales contextualizados. Siguiendo esa premisa, el artículo analiza, por un lado, la evolución y los resultados de las investigaciones realizadas en los últimos años sobre la transición a la universidad de los estudiantes en el sistema universitario catalán y, específicamente, de la Universidad de Barcelona; y por otro lado, presenta la última investigación desarrollada en dos ámbitos disciplinarios distintos de Ciencias Sociales: Administración y Dirección de Empresas (ADE) y Pedagogía[1]. Los resultados permiten contextualizar las dificultades en el proceso de transición y la importancia significativa de la adaptación académica en el primer año de estudios de manera segmentada. Por último, enfoca la necesidad de revisar las medidas de retención establecidas en la universidad, incluyendo las acciones de tutoría, con la finalidad de optimizar la persistencia de los estudiantes.

PALABRAS CLAVE: Estudiantes universitarios. Adaptación académica. Persistencia.

\section{LAS ENSEÑANZAS DE UNA DÉCADA DE INVESTIGACIONES SOBRE LA PERSISTENCIA/ABANDONO EN EL SISTEMA UNIVERSITARIO DE CATALUÑA}

La preocupación por la transición a la Universidad no es un problema nuevo en nuestro país. Son numerosos los estudios que se han realizado en estos últimos treinta años. Muchos de ellos centrados en contextos de enseñanza a distancia o en titulaciones con elevadas tasas de abandono; la mayoría con enfoques globalizadores y cuantitativos.

En esta última década las investigaciones relacionadas con la persistencia y el abandono universitario han centrado su interés en conocer con mayor profundidad algunos factores asociados al fenómeno, con el objetivo de poder contribuir a la construcción de un modelo explicativo de la transición. Desde una perspectiva metodológica también se puede constatar una evolución. Así, una parte importante de la investigación desarrollada en el campo del abandono-persistencia universitaria se ha basado en estudios descriptivos con grandes muestras de datos; sin embargo, la constatación de la complejidad y multidimensionalidad del fenómeno ha impulsado, más recientemente, planteamientos metodológicos complementarios que incluyen el uso de metodologías de carácter cualitativo dirigidas a comprender las dinámicas implícitas en los procesos de transición a la universidad y a diseñar estudios contextualizados en las diferentes realidades. Se entenderán, así, los factores que afectan la toma de decisión y se podrán aplicar metodologías que permitan - en ese primer año de transición - un seguimiento de los estudiantes para identificar sus decisiones o intenciones de abandono (riesgo), así como los factores que intervienen en la consolidación de la decisión de abandonar o persistir. Las investigaciones recientes como las de Castaño y otros (2008) señalan, además, el valor de este tipo de estudios para analizar las variables que evolucionan con el tiempo (integración académica y social, aspectos institucionales, dedicación, etc.)

En el caso concreto de Cataluña, los primeros estudios amplios se inician a finales de la década de los 90 liderados por el equipo de investigación TRALS[2] de la Universidad de Barcelona. En una primera línea de trabajos se analizaron los factores estructurales de la transición, incluyendo 
la incidencia de la reforma educativa LOGSE[3] sobre el rendimiento académico universitario. Algunas conclusiones de estos primeros estudios fueron: la variable género como factor no diferenciador en el contexto general, pero sí en contextos específicos; el rendimiento previo y la nota de acceso como claros predictores del rendimiento académico en los dos primeros años de carrera (FIGUERA y TORRADO, 2000; DE MIGUEL, RODRÍGUEZ et al., 2005; RODRÍGUEZ, FITA y TORRADO, 2004). La insuficiencia explicativa de los factores estructurales de acceso a la universidad para explicar los logros de la transición Secundaria-Universidad llevó a plantear, en una segunda línea de estudios, la revisión de los indicadores y la propuesta de un modelo más comprensivo, basado en el modelo de Vicent Tinto. Se incluyeron como otros indicadores clave: la satisfacción de la propia experiencia universitaria, el desarrollo personal del estudiante y la adquisición y desarrollo de las habilidades de gestión personal. Los resultados evidenciaron el importante aporte que constituye la experiencia académica previa del alumno para resolver satisfactoriamente su transición a la universidad, así como el valor del apoyo familiar tanto para las expectativas de logro como para la motivación hacia los estudios universitarios (FIGUERA et al., 2003), resultados coherentes con trabajos de Cabrera, Nora y Castaneda (1993) en el contexto norteamericano. También se comprobaron la importancia de la información y la orientación previas a la transición a la universidad como mecanismos de prevención del fracaso o frustración de las expectativas iniciales (ÁLVAREZ y FITA, 2005; ÁLVAREZ, FIGUERA y TORRADO, 2011).

En el tránsito al siglo XXI, el reto de Bolonia exige cambios radicales en los procesos de enseñanza-aprendizaje y en los sistemas organizativos universitarios, que han de adaptarse a nuevos objetivos. Uno de ellos es la dimensión social de la educación, que se refiere a que el acceso a la Universidad y la trayectoria a seguir, así como los resultados finales, sean inclusivos al basarse en la equidad participativa. Eso significa considerar dos dimensiones: la diversidad de estudiantes y los resultados finales entendidos como persistencia y abandono.

En relación a la incidencia del abandono en el contexto universitario, el estudio realizado, en colaboración con otros equipos, por miembros del grupo TRALS, señala la pervivencia de altas tasas de abandono en el contexto de las universidades públicas catalanas, y la importancia del primer año en la decisión de abandonar (GAIRÍN, FIGUERA y TRIADÓ, 2010). Las tasas de abandono en Cataluña ascienden a un $34,1 \%$; las mayores tasas de abandono se concentran en las carreras del ámbito de ciencias sociales $(40 \%)$, seguidas de las titulaciones del área técnica $(29,6 \%)$ y humanidades $(17,2 \%)$. El fenómeno afecta de manera significativa a todas las universidades catalanas y a todas las titulaciones. Las variables edad, género, nota de acceso y dedicación mostraron relación significativa con el abandono. Otras conclusiones apuntan a la incidencia de la motivación sobre la integración académica al decrecer la asistencia a clase y a las actividades sociales, aspectos también destacados en los trabajos recientes del equipo GUFOI[4] en el contexto universitario Canario (MARTIN y otros, 2010).

Las diferencias entre las áreas de estudio apuntan como primera hipótesis que los ámbitos disciplinares y las titulaciones son un factor clave para un adecuado análisis de las trayectorias académicas. En esta línea de investigaciones más contextualizadas, la tesis doctoral de M. Torrado (2012), del equipo TRALS, ha permitido valorar, en un estudio longitudinal amplio, la caracterización y significado de las trayectorias de postabandono en las Ciencias Experimentales en la UB. Los resultados de este trabajo cuestionan la interpretación del fenómeno del abandono, ya que el seguimiento longitudinal de los estudiantes en diez años confirmó que casi la totalidad de los que abandonaron la carrera inicial reingresaron con éxito en otras carreras.

Por último, y desde una perspectiva centrada en colectivos minoritarios, los resultados del estudio de la transición a la universidad de las personas con discapacidad (FIGUERA y otros, 2010) ponen de manifiesto la existencia de barreras institucionales y personales con un peso diferencial a la población general, cuya comprensión requiere la importancia de identificar precozmente los colectivos con un alto riesgo de abandono.

\section{FACTORES DE ADAPTACIÓN Y PERSISTENCIA UNIVERSITARIA}

En el campo de estudio se considera el modelo teórico explicativo de V. Tinto, formulado inicialmente en la década de los 70 y sometido a desarrollos posteriores, como uno de los más significativos. Tinto propone una estructura fuerte, de gran consistencia, que ayuda a entender los factores que afectan las decisiones de abandono o persistencia de los estudiantes universitarios. 
Para él, la integración académica y social es la explicación del por qué un alumno se adapta y permanece en el sistema fidelizándose a la institución. La permanencia en el sistema está influida por el bagaje de entrada del alumno, sus antecedentes académicos previos o preparación de base, el entorno familiar y las características personales, el compromiso inicial con la institución, la intención de finalizar los estudios y las interacciones positivas con el entorno. En sus últimas aportaciones el modelo incorpora al papel del esfuerzo del estudiante y el aprendizaje como eje fundamental para el éxito académico y le da un valor añadido a la institución (soporte social, el clima, la cultura de grupo, las interacciones con profesores y compañeros,...),

Completando el modelo integrador de Tinto, el enfoque sociocognitivo presenta un marco conceptual idóneo para entender y profundizar sobre la interacción de los factores sociocognitivos que participan en el proceso de ajuste académico y en la satisfacción del estudiante en ámbitos académicos. Dentro de este enfoque, una de las formulaciones más emergentes es el modelo de ajuste psicosocial y satisfacción académica de Robert W. Lent (2004), utilizado en nuestros estudios como referente teórico. Según el modelo, las expectativas de autoeficacia y el soporte social son dos factores explicativos clave al actuar directamente sobre los diversos indicadores de integración académica y, a la vez, indirectamente a través de la influencia sobre la variable actividad hacia la meta, precursora de la integración (LENT y otros, 2009).

Apoyados en estos modelos, diversas investigaciones han ahondado en el valor predictivo de los factores personales y contextuales en las trayectorias de retención. Los resultados de estos estudios confirman que las variables demográficas (edad, género, etnia, situación laboral y financiera) se asocian a trayectorias con un mayor riesgo de abandono (BOWEN y otros, 2009; CRAWFORD y HARRIS, 2008; CABRERA y otros, en prensa). Igualmente, las variables sociocognitivas, como la autoestima, el estrés, la autoeficacia, se consideran básicas en la explicación de trayectorias de continuidad (OJEDA y otros, 2011; HONG y otros, 2012). También las variables que conforman la experiencia académica previa (centro de estudios, rendimiento, hábitos de estudio, vía de acceso a la universidad, apoyo académico, competencias previas) se muestran relevantes, especialmente, en el primer año de universidad (YORKE y LONGDEN, 2008).

El estudio de la influencia de los contextos en que participa el estudiante (familiar, institucional y social) aporta interesantes hallazgos en la resolución de la transición. El apoyo familiar es una variable muy significativa en la explicación de la integración académica y social del estudiante. Los estudios confirman la tesis de que los estudiantes universitarios con una alta percepción de soporte familiar confían más en su capacidad para superar los objetivos académicos, tienen mayores niveles de autoeficacia académica y son más autónomos académica y socialmente (ROWAN-KENYON y otros, 2008). En cambio, cuando la familia no puede asegurar los recursos necesarios para sufragar el coste universitario existe riego de abandono (NORA y CRISP, en prensa). Por otro lado, el apoyo institucional es clave para la retención de los estudiantes cuando estimula el desarrollo académico, clarificando objetivos y demandas académicas; orienta sobre cómo conocer y adaptarse al contexto universitario y, además, crea espacios para que el estudiante pueda ser un miembro activo de la comunidad universitaria (KARP, 2011; SCOTT-CLAYTON, 2011).

Se identifican otros factores institucionales que explican la persistencia universitaria: las características de las enseñanzas de la institución, las ayudas pedagógicas y de orientación, la demanda de trabajo exigido, la calidad del profesorado o el clima en las aulas (TINTO, 2010). En relación a la actividad académica, es significativa la relación con la utilización del tiempo y los hábitos de estudio (NONIS y HUDSON, 2010) y la relación entre el profesorado y el estudiante (LILLIS, 2012). Finalmente, el soporte social de los compañeros y amigos, así como las acciones institucionales para fomentar y facilitar el establecimiento de relaciones sociales en los estudiantes al inicio de su vida en la universidad, contribuyen de manera significativa a incrementar los recursos personales de afrontamiento de la transición (STRYDOM y MENTZ, 2010; KARP, 2011). Los autores confirman la necesidad de considerar la interacción de estos factores en contextos disciplinares específicos, como el estudio desarrollado en el marco de la Universidad de Barcelona por el equipo TRALS.

\section{METODOLOGÍA DE LA INVESTIGACIÓN}

El estudio forma parte de una investigación desarrollada por TRALS en el marco del proyecto I+D: "La persistencia y el abandono en el primer año de universidad en ciencias sociales: bases para la 
mejora de la retención" cuya finalidad es describir y comprender los factores personales e institucionales de persistencia académica al finalizar el primer año de universidad en las carreras de Ciencias Sociales. Más específicamente, se ha llevado a cabo un estudio longitudinal de la cohorte 2010 de alumnos que accedieron a los nuevos Grados de "Administración y Dirección de Empresa" (ADE) y "Pedagogía" de la Universidad de Barcelona. El total de participantes ha sido: ADE ( $N=1290)$ y Pedagogía $(N=233)$.

La investigación parte de la hipótesis que la persistencia y el abandono universitario responden a una interacción compleja de factores de carácter institucional y personal, cuya comprensión exige un análisis longitudinal y contextualizado en ámbitos disciplinares específicos (carreras) que permitan una adecuada comprensión de los procesos de transición de los estudiantes y de los factores intervinientes.

El estudio se desarrolló en tres momentos a lo largo del primer año: a los dos meses de iniciar los estudios; al inicio del segundo semestre, una vez conocidos los resultados del primer semestre y las demandas de las asignaturas del segundo semestre y al inicio del segundo año. Las diversas fuentes de información utilizadas han permitido un acercamiento más global (Macro) y otro más específico (Micro) de la persistencia del primer año de estos estudiantes. Desde la mirada del estudiante se han combinado los datos institucionales (personales y académicos) de cada alumno, tres cuestionarios de aplicación en el aula para recoger evidencias directamente del estudiante (sobre el ajuste y adaptación académica, percepción de apoyo recibido, expectativas de autoeficacia, satisfacción académica, etc.) y dos grupos de discusión al final de cada semestre. Y desde el punto de vista de la institución, el análisis de las variables institucionales (características de los dos grados -planificación del currículum, desarrollo del proceso de enseñanza, perfil del profesorado, etc.-) y la visión del docente de las asignaturas del primer año (actitudes y conductas del grupo clase y conocer la opinión del contexto docente) han permitido complementar el marco contextual de estos estudiantes.

\section{RESULTADOS}

Los resultados obtenidos apuntan hacia una constatación clara de la existencia de dos microsistemas diferentes en cuanto a la concepción, organización y desarrollo del proceso de enseñanza-aprendizaje, variables que condicionan, sin duda, el proceso de adaptación y facilitan o inhiben la integración académica y las posibilidades de interacción social de los estudiantes. Así, la concepción de la enseñanza del profesorado de ADE y de Pedagogía difiere en aspectos significativos como: el modelo de enseñanza y de tutoría, la relación con el estudiante y la importancia dada al clima de clase como factor favorecedor de la integración y el rendimiento del estudiante. Esto se transfiere en la realidad cuando se observa una mayor diversidad y uso de estrategias de enseñanzaaprendizaje en Pedagogía, trabajo en grupo y papel activo del estudiante, frente a un enfoque más tradicional entre el profesorado de ADE.

El perfil de los estudiantes de ambas titulaciones es diferente, significativamente, en cuanto al sexo (89\% de mujeres en Pedagogía), en el background académico previo (nota de acceso mayor en $A D E$ ) y en la calidad de la decisión inicial (opción de acceso). En cuanto a los indicadores de ajuste inicial (satisfacción académica, adaptación, expectativas y motivación) fueron significativamente inferiores en los estudiantes de $A D E$, considerados en conjunto, frente a los estudiantes de Pedagogía. Estas diferencias no se relacionan con el perfil de entrada de los estudiantes, sino con las variables del contexto institucional (clima, estrategias de enseñanza, relación profesorado, soporte, dificultad estudios). El análisis segmentado por carrera confirma la asociación entre las diversas variables sociocognitivas analizadas: expectativas de autoeficacia, el apoyo académico y el apoyo social y familiar.

Tras el primer semestre se constata la incidencia de la dificultad y el nivel de exigencia en los estudios y la importancia de la contextualización. El análisis segmentado confirma la relación de la nota media con la conducta hacia el estudio y autoeficacia académica. Los indicadores de adaptación académica (satisfacción, adaptación, expectativas y motivación) son significativamente inferiores en los estudiantes de ADE, independientemente de la vía de acceso. La predicción de la satisfacción académica al final del primer semestre está directamente relacionada, en ADE, con la percepción de afrontamiento de las demandas académicas. En una y otra titulación los resultados de los grupos de discusión confirman la influencia del "clima de aula" y la interacción con los iguales como factores de ayuda en la adaptación a la universidad. 
En el inicio del segundo año, los estudiantes que persisten presentan niveles similares de motivación pero difieren en relación a los indicadores de integración académica y social, en la misma dirección que en las fases anteriores. Los estudiantes de Pedagogía se sienten más satisfechos con el ambiente educativo y el contexto social y confirman un mayor nivel de estimulación académica que sus compañeros de ADE. La vía de acceso presenta diferencias significativas en estas variables, reafirmándose niveles más altos en la conducta de gestión del estudio entre el alumnado procedente del bachillerato.

Independientemente de la carrera, los estudiantes señalan como aspectos fundamentales del éxito en la transición la asistencia continuada y activa en la clase y una conducta y gestión del estudio de manera más constante y responsable.

A partir de los grupos de discusión, los estudiantes han identificado los tres momentos más críticos en el desarrollo académico en el primer año y, por lo tanto, más sensibles a la decisión de abandonar. El primer período es el de las primeras semanas de clase en las que el estudiante realiza un primer contraste entre la información inicial, sus expectativas y la realidad de los estudios, afectando en la rectificación de sus expectativas de autoeficacia y su motivación. El segundo período sensible es el del "rendimiento de cuentas" que se ve afectado, como se ha podido constatar, por el modelo de evaluación predominante y en el que la capacidad de gestionar el estrés y el apoyo de los iguales es clave. Y como tercer período clave estaría el posterior al conocimiento de las calificaciones del primer semestre (contraste de autoeficacia). Los indicadores de satisfacción académica y expectativas de autoeficacia presentan valores más constantes pasados estos períodos críticos.

Uno de los factores clave de la transición es la orientación y la atención tutorial del estudiante. Una función en la que deben estar implicados los centros de procedencia y los centros de recepción. Los resultados de los estudios ofrecen algunas evidencias sobre la adecuación de los sistemas actuales de soporte al estudiante en su adaptación inicial.

Respecto al impacto en los procesos de adaptación e integración inicial en la universidad a partir del estudio en profundidad de las titulaciones de ADE y Pedagogía se constata, en primer lugar, una ausencia de coordinación entre los sistemas precedentes y de destino, es decir, entre el sistema de secundaria y la universidad. La labor de orientación para la elección de estudios en secundaria queda en manos, en muchos centros educativos, del tutor/a, sin apoyo de un profesional formado que pueda dar apoyo a la labor tutorial.

En ambos contextos disciplinares, la información al alumnado de nuevo acceso se centra en la web como el canal básico de comunicación. Aunque los estudiantes la conocen y la han utilizado con cierta asiduidad, se han destacado problemas de accesibilidad y usabilidad (comprensión) que pueden incidir indirectamente en la calidad de la decisión y en los procesos de integración inicial.

En cuanto a la incidencia de las acciones de orientación y tutoría en los centros universitarios, las aportaciones de los grupos de discusión han ayudado a constatar el gran desconocimiento por parte de los alumnos del sentido y finalidad de los planes de acción tutorial. En ambas titulaciones aparecen disfunciones en cuanto a la implementación de estas acciones y no empiezan a ser efectivas hasta que no ha transcurrido un semestre, una vez superados los períodos críticos señalados. Este hecho ya constatado por Dorio, Peró y Torrado (2005) cuestiona la efectividad y eficacia de estos planteamientos, no tanto en su diseño sino en su implementación en las aulas.

\section{CONCLUSIONES Y PROSPECTIVA}

La investigación desarrollada permite afirmar que las trayectorias finales son, como indican las investigaciones, producto de una interacción a lo largo del tiempo de factores institucionales y factores personales. El análisis segmentado de la multidimensionalidad del fenómeno de la persistencia en dos entornos organizativos diferentes de una misma universidad confirma la influencia del contexto en la interpretación de la transición académica en el primer año de carrera. El peso explicativo de los factores estudiados difiere según el contexto analizado. No obstante, y en función de la permanencia o abandono en la universidad, e independientemente del contexto, aparece un patrón común en cuanto a aspectos clave como son: los momentos críticos donde el alumno llega a plantearse "el deseo de abandonar", las características personales (edad, dedicación al estudio,...) que identifican 
colectivos de riesgo de no permanencia y la importancia de las acciones de orientación e información en la universidad como facilitadoras de la adaptación académica.

A la luz de los resultados obtenidos se reconoce la necesidad de investigar la relación entre persistencia en el primer año y graduación, así como profundizar en los factores determinantes de la persistencia o abandono en años posteriores al primer año. El trabajo pionero de Nora y Crips (en prensa) en EEUU, y Torrado (2012) en España, confirman perfiles diferenciales en las trayectorias de abandono cuando se considera el tiempo o año de abandono. Es razonable suponer que la fuerza y la dirección de los factores que influyen en el comportamiento de la persistencia pueden cambiar con el tiempo (ISHITANI, 2008). Sin embargo, y a tenor de la revisión de la literatura, podemos afirmar que existen pocas investigaciones centradas en la identificación de las variables que influyen en las decisiones de los estudiantes más allá de la persistencia del primer año, en la configuración de las trayectorias y en el logro de la graduación.

\section{REFERENCIAS}

ÁLVAREZ, M. y FITA, E. (2005). La intervención orientadora en la transición bachilleratouniversidad. Bordón, 57, pp. 5-27.

ÁlVAREZ, M., FIGUERA, P. y TORRADO, M. (2011). La problemática de la transición bachilleratouniversidad en la Universidad de Barcelona. Revista Española de Orientación Psicopedagógica, 22, pp. 15-27.

BOWEN, W. G., CHINGOS, M. M., y MCPHERSON, M. S. (2009). Crossing the finish line: Completing college at America's public universities. Princeton, NJ: Princeton University Press.

CABRERA, A.; NORA, A. y CASTANEDA, M. (1993). Structural equations modelling test o fan integrated model of student retention. Journal of Higher Education, 64, pp. 123-139.

CABRERA, A., BURKUM, K.R., LANASA, S.M. y BIBO, E.W. (en prensa) Pathways to a Four-Year Degree: Determinants of Transfer and Degree Completion among Socioeconomically Disadvantaged Students. En A. Seidman (Ed.), College student retention. Westport, CT: Praeger Publishers.

CASTAÑO, E., GALLÓN, S., GÓMEZ, K. y VÁZQUEZ, J. (2008). Análisis de los factores asociados a la deserción estudiantil en la Educación Superior. Revista de Educación, 345, pp. 255-280.

CRAWFORD, K. y HARRIS, M. (2008). Differential predictors of persistence between community college adult and traditional aged students. Community College Journal of Research and Practice, 32 , pp. 75-100.

DE MIGUEL, M.; APODAKA, P., ARIAS, J. M.; ESCUDERO, T.; RODRÍGUEZ, S. y VIDAL, J. (2005). To what extent is higher education achievement conditioned by the secondary education model. Studies in Education Evaluation, 31, pp. 57-78.

DORIO, I.; PERÓ, M. y TORRADO, M. (2005). Avaluació dels plans d'acció tutorial a la Universitat de Barcelona. Informe Global (documento inédito de la UB).

FIGUERA, P. Y TORRADO, M. (2000) El proceso de transición del bachillerato a la universidad: factores de éxito. Quaderns Institucionals, 2, pp. 41-55.

FIGUERA, P.; DORIO, I. Y FORNER, A. (2003). Las competencias académicas previas y el apoyo familiar en la transición a la universidad. Revista de Investigación Educativa, 21, pp. 349-369.

FIGUERA, P., (DIR.), COIDURA, J., FREIXA, M., ISÚS, S., RODRÍGUEZ, M.L. y TORRADO, M. (2010). La transición a la universidad de las personas con discapacidad. Madrid: Dirección General de Universidades. Programa de Estudios y Análisis 2009.

FIGUERA, P., TORRADO, M., POL, C. y CORTI, F. (2011). Análisis del perfil del estudiante y del proceso de integración académica en la universidad. Implicaciones para la relación pedagógica. En F. Hernández (coord.) Pensar la relación pedagógica en la universidad desde el encuentro entre sujetos, deseos y saberes. Barcelona: Universitat de Barcelona. http://hdl.handle.net/2445/20946.

GAIRÍN, J.; FIGUERA, P. y TRIADÓ, X. (Coord.) (2010). L'abandonament dels estudiants a les universitats catalanes. Barcelona: Agència de Qualitat del Sistema Universitari de Catalunya (AQU). 
HONG, B.S.; SHULL, P.J. y HAEFNER, L. A. (2012). Impact of perceptions of faculty on student outcomes of self-efficacy, locus of control, persistence, and commitment. Journal of College Student Retention: Research, Theory \& Practice, 13, pp. 289-309.

ISHITANI, T.T. (2008). How to explore timing of intervention for student at risk of departure. In: T.T. Ishitani (Ed.) Alternative perspectives in institutional planning: New directions for institutional research. Jossey-Bass.

KARP, M.M. (2011). How non-academic supports work: four mechanisms for improving student outcomes. Community College Research Center, Columbia University.

LENT, R. W. (2004). Toward a unifying theoretical and practical perspective on well-being and psychosocial adjustement. Journal of Counseling Psychology, 51, pp. 482-509.

LENT, R. W. ; TAVEIRA, M. D. ; SHEU, H. y SINGLEY, D. (2009). Social cognitive predictors of academic adjustment and life satisfaction in portugues college students: a longitudinal analysis. Journal of Vocational Behavior, 74, pp. 190-198.

LILLIS, M. P. (2012). Faculty emotional intelligence and student-faculty interactions: implications for student retention. Journal of College Student Retention: Research, Theory \& Practice, 13, pp. 155-178.

MARTÍN, E.; GONZÁleZ, M.; RODRÍGUeZ, J.; PÉREZ, C. ; ÁlVAREZ, P. y COLS. (2010). El rendimiento académico del alumnado de nuevo ingreso en la Universidad de La Laguna. La Laguna, Servicio de Publicaciones de la Universidad de La Laguna.

NONIS, S. y HUDSON, G. I. (2010). Performance of college students: Impact of study time and study habits. Journal of Education for Business, 85, pp. 229-238.

NORA, A. y CRIPS, G. (en prensa). Student persistence and degree attainment beyond the first year in college: Existing knowledge and directions for future research. En A. Seidman (ed). College student retention. Formula for student success. Rowman \& Littlefield publishers, inc. 2 edición.

OJEDA, L., NAVARRO, R. y FLORES, L. (2011). Social cognitive predictors of mexican collage students academic and life satisfaction. Journal of Counseling Psychology, 58, pp. 61-71.

RODRÍGUEZ, S.; FITA, E. y TORRADO, M. (2004). El rendimiento académico en la transición secundaria-universidad. Revista de Educación, 334, pp. 391-414.

ROWAN-KENYON, H. T.; BELL, A. D. y PERNA, L. W. (2008). Contextual influences on parental involvement in college going: variations by socioeconomic class. The Journal of Higher education, 79, pp. 564-586.

SCOTT-CLAYTON, J. (2011). The shapeless river: does a lack of structure inhibit students' progress at community colleges? CCRC working paper, n० 25.

STRYDOM, J.F. y METNTZ, M (2010). Focusing the student experience on success through student engagement. Pretoria: Council on Higher Education.

TINTO, V. (2010). From theory to action: Exploring the institutional conditions for student retention. Higher Education: Handbook of Theory and Research. Vol. XXV, pp. 51-90.

TORRADO, M. (2012). El fenómeno del abandono en la Universidad de Barcelona: el caso de ciencias experimentales. Universidad de Barcelona. Tesis doctoral inédita.

YORKE, M. y LONGDEN, B. (2008). The first-year experience of higher education in the UK. York, UK: The Higher Education Academy.

NOTAS

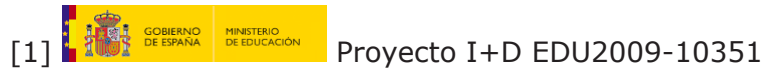

[2] TRALS (Transiciones académicas y laborales). Equipo de investigación coordinado por la Dra. Pilar Figuera del departamento de Métodos de Investigación y Diagnóstico en Educación (MIDE) de la Universidad de Barcelona (http://www.ub.wedu/trals). 
[3] La LOGSE (Ley Orgánica General del Sistema Educativo) fue una ley educativa aprobada el 3 de octubre 1990 y que sustituyó la Ley General de Educación de 1970. En 2006 se aprobó la LOE (Ley Orgánica de Educación).

[4] GUGOI (Grupo Universitario de formación y orientación integrada). Es un grupo de investigación de la Universidad de La Laguna coordinado por el Dr. Pedro R. Álvarez. (http://www.gufoi.ull.es/).

Artigo recebido em 23/01/2013

Aprovado em 07/02/2013 